\title{
ALGEBRAIC VARIETIES ARE HOMEOMORPHIC TO VARIETIES DEFINED OVER NUMBER FIELDS
}

\author{
ADAM PARUSIŃSKI AND GUILLAUME ROND
}

\begin{abstract}
We show that every affine or projective algebraic variety defined over the field of real or complex numbers is homeomorphic to a variety defined over the field of algebraic numbers. We construct such a homeomorphism by carefully choosing a small deformation of the coefficients of the original equations. This deformation preserves all polynomial relations over $\mathbb{Q}$ satisfied by these coefficients and is equisingular in the sense of Zariski.

Moreover we construct an algorithm, that, given a system of equations defining a variety $V$, produces a system of equations with coefficients in $\overline{\mathbb{Q}}$ of a variety homeomorphic to $V$.
\end{abstract}

\section{INTRODUCTION}

In computational algebraic geometry one is interested in computing the solution set of a given system of polynomial equations, or at least in computing various algebraic or geometric invariants of such a solution set. Here by computing we mean producing an algorithm that gives the desired result when it is implemented in an appropriate computer system.

Such an algorithm should be stable with respect to a small perturbation of the coefficients. But in general the shape of the solution set may change drastically under a small perturbation of the coefficients. This difficulty is particularly apparent if one has to deal with polynomial equations whose coefficients are neither rational nor algebraic numbers. The main goal of this paper is to show that, when we are interested in the topology of such a solution set, one can always assume that the polynomial equations have algebraic number coefficients precisely by choosing in an effective way a particular perturbation of its coefficients.

Theorem 1. Let $V \subset \mathbb{K}^{n}$ (resp. $V \subset \mathbb{P}_{\mathbb{K}}^{n}$ ) be an affine (resp. projective) algebraic set, where $\mathbb{K}=\mathbb{R}$ or $\mathbb{C}$. Then there exist an affine (resp. projective) algebraic set $W \subset \mathbb{K}^{n}$ (resp. $W \subset \mathbb{P}_{\mathbb{K}}^{n}$ ) and a homeomorphism $h: \mathbb{K}^{n} \longrightarrow \mathbb{K}^{n}$ (resp. $\left.h: \mathbb{P}_{\mathbb{K}}^{n} \longrightarrow \mathbb{P}_{\mathbb{K}}^{n}\right)$ such that:

(i) the homeomorphism maps $V$ onto $W$,

(ii) $W$ is defined by polynomial equations with coefficients in $\overline{\mathbb{Q}} \cap \mathbb{K}$.

(iii) The homeomorphism $h$ is semialgebraic and arc-analytic.

Arc-analytic semialgebraic homeomorphisms play an important role in real algebraic geometry, see Remark 12 .

Remark 2. In fact we prove a more precise and stronger result. See Theorem 11 for a precise statement.

Research partially supported by ANR project LISA (ANR-17-CE40-0023-03). 
In the proof of Theorem 11 we show that $W$ can obtained by a small deformation of the coefficients of the equations defining $V$. Let denote these coefficients by $g_{i, \alpha}$. The deformation is denoted by $t \mapsto g_{i, \alpha}(t)$ with $g_{i, \alpha}(0)=g_{i, \alpha}$. This deformation is constructed in such a way that every polynomial relation with rational coefficients satisfied by the $g_{i, \alpha}$ is satisfied by the $g_{i, \alpha}(t)$ for every $t$, c.f. Theorem 4 . So we can prove that the deformation is equisingular in the sense of Zariski since this equisingularity condition can be encoded in term of the vanishing and the nonvanishing of polynomial relations on the $g_{i, \alpha}(t)$. In particular this deformation is topologically trivial.

The first part of this paper is devoted to the proof of Theorem 4 , an approximation result similar to the one given in $\underline{\mathrm{RO}}$. In the next part we recall the notion of Zariski equisingularity. The proof of Theorem 11 is given in the following part. Finally we provide an algorithm that, given the equations defining $V$, computes the equations defining $W$. This algorithm is based on the proof of Theorem 11

1.1. Topologically trivial stratifications. Theorem 1 can be also proven by the following general argument. Denote, as above, the coefficients of the polynomials $F_{1}, \ldots, F_{r}$ defining $V$ by $g_{i, \alpha}$ and denote their number by $N$. Let $\mathcal{V} \subset \mathbb{K}^{N} \times \mathbb{K}^{n}$ be the zero set of $F_{1}, \ldots, F_{r}$, where we consider their coefficients as parameters $\left(c_{i, \alpha}\right) \in \mathbb{K}^{N}$. Thus $V$ is the fiber of $\mathcal{V}$ over $\left(g_{i, \alpha}\right)$. There exists a stratification $\mathcal{S}$ of the coefficients space $\mathbb{K}^{N}$ such that the fibres of $\mathcal{V}$ are locally topologically trivial over each stratum. Such a stratification can be, for instance, constructed by Zariski equisingularity, and in this case, by construction, each stratum $S$ of $\mathcal{S}$ is described by polynomial equations and inequalities in $c_{i, \alpha}$ with coefficients in $\mathbb{Q}$. Therefore, Theorem 1 follows from the fact that $\overline{\mathbb{Q}} \cap S$ is dense in $S$ (this follows, for instance, from our Theorem (4). It seems that this argument was known to specialists in equisingularity theory, though we couldn't have found it in literature.

In the real algebraic (or semialgebraic) set-up one can put the above argument in terms of Tarski's transfer principle, cf. BCR Ch. 5. More precisely, let us first work over the real closed field of algebraic numbers $\mathbb{R}_{\text {alg }}=\overline{\mathbb{Q}} \cap \mathbb{R}$. By Hardt's trivialization theorem [H80] there is a stratification of $\mathcal{S}_{\text {alg }}$ of $\mathbb{R}_{\text {alg }}^{N}$ that trivializes $\mathcal{V}$. Moreover, the trivialization maps are semialgebraic. By Tarski's transfer principle both the stratification and the trivializations extend to $\mathbb{R}$, that is the same equations and inequalities give a stratification $\mathcal{S}$ of $\mathbb{R}^{N}$, that is locally trivial with the trivializations given exactly by the same formulae.

Our approach is slightly different since we do not stratify the coefficient space. Instead, as we explained above, we produce a deformation of the coefficients, that preserves polynomial relations with rational coefficients and therefore is topologically trivial. For the last argument we use Zariski equisingularity, since it works over both $\mathbb{R}$ and $\mathbb{C}$, and gives trivialization with many additional properties (including semialgebraicity). This deformation is based directly on the properties of the field extension of $\mathbb{Q}$ generated by the coefficients $g_{i, \alpha}$. For instance, it is particularly simple if this extension is purely transcendental, see Remark 13.

Acknowledgements. We would like to thank an anonymous referee for drawing our attention to the role of transfer principle in this context.

Notation. For a vector of indeterminates $x=\left(x_{1}, \ldots, x_{n}\right), x^{i}$ denotes the vector of indeterminates $\left(x_{1}, \ldots, x_{i}\right)$. To avoid confusion, variables will be denoted by 
normal letters $t$ and elements of $\mathbb{K}$ will be denoted by bold letters $\mathbf{t}$. For a field $\mathbb{K}$ the ring of algebraic power series is denoted by $\mathbb{K}\langle x\rangle$.

\section{An APPROXimation RESUlt}

Let $\Omega \subset \mathbb{K}^{r}$ be open and non-empty and let $\varphi$ be an analytic function on $\Omega$. We say that $\varphi$ is a Nash function at $a \in \Omega$ if there exist an open neighborhood $U$ of $a$ in $\Omega$ and a nonzero polynomial $P \in \mathbb{K}\left[t_{1}, \ldots, t_{r}, z\right]$ such that $P(\mathbf{t}, \varphi(\mathbf{t}))=0$ for $\mathbf{t} \in U$ or, equivalently, if the Taylor series of $\varphi$ at $a$ is an algebraic power series. An analytic function on $\Omega$ is a Nash function if it is a Nash function at every point of $\Omega$. An analytic mapping $\varphi: \Omega \rightarrow \mathbb{K}^{N}$ is a Nash mapping if each of its components is a Nash function. Note that if $\Omega$ is connected and $\varphi$ is Nash then, by analytic continuation, it satisfies one polynomial equation $P(\mathbf{t}, \varphi(\mathbf{t}))=0$ everywhere on $\Omega$.

We call a Nash function $\varphi: \Omega \rightarrow \mathbb{K}, \mathbb{Q}$-algebraic (and we note $\varphi \in \mathrm{QA}(\Omega)$ ) if (locally) it is algebraic over $\mathbb{Q}[t]$, i.e. it satisfies a polynomial relation $P(\mathbf{t}, \varphi(\mathbf{t}))=0$ for every t $\in \Omega$ with $P \in \mathbb{Q}\left[t_{1}, \ldots, t_{r}, z\right], P \neq 0$. It is well known, in the case when $\Omega$ is connected, that this means that the Taylor expansion of $\varphi$ at a point with coordinates in $\mathbb{Q}$ (or $\overline{\mathbb{Q}}$ ) is an algebraic power series whose coefficients lie in a common finite field extension of $\mathbb{Q}$, i.e. this Taylor expansion belongs to $\mathbb{k}\langle x\rangle$ where $\mathbb{k}$ is a subfield of $\mathbb{K}$ which is finite over $\mathbb{Q}$ (see [RD84 for example).

Remark 3. Usually, Nash functions are defined as real analytic functions defined on an open set $\Omega \subset \mathbb{R}^{r}$ whose graph is semialgebraic. In particular, $\Omega$ has to be semialgebraic. Here, we need a definition valid both for $\mathbb{K}=\mathbb{R}$ and $\mathbb{C}$. Moreover, in practice, the open set $\Omega$ will always be connected, and one may choose $\Omega$ semialgebraic. Nash functions, as we defined above, are used sometimes in complex algebraic geometry, see for instance L95. Therefore, we have chosen a simple definition, that is slightly more general than the usual one in the real case, but easier to handle when we consider simultaneously the cases $\mathbb{K}=\mathbb{R}$ and $\mathbb{C}$.

Theorem 4. Let $\mathbb{K}=\mathbb{R}$ or $\mathbb{C}$. Let $\mathbf{y} \in \mathbb{K}^{m} \backslash \overline{\mathbb{Q}}^{m}$. Denote by $\mathbb{k}$ the field extension of $\mathbb{Q}$ generated by the components $\mathbf{y}_{i}$ of $\mathbf{y}$. The field $\mathbb{k}$ is a primitive extension of a transcendental finitely generated field extension of $\mathbb{Q}: \mathbb{k}=\mathbb{Q}\left(\mathbf{t}_{1}, \ldots, \mathbf{t}_{r}\right)(\mathbf{z})$ where the $\mathbf{t}_{i} \in \mathbb{K}$ are algebraically independent over $\mathbb{Q}$ and $\mathbf{z} \in \mathbb{K}$ is finite of degree $d$ over $\mathbb{Q}\left(\mathbf{t}_{1}, \ldots, \mathbf{t}_{r}\right)$.

Then there exist an open, connected and non-empty neighborhood of $\mathbf{t}=\left(\mathbf{t}_{1}, \ldots, \mathbf{t}_{r}\right)$, $\mathcal{U} \subset \mathbb{K}^{r}$, a vector function well defined on $\mathcal{U} \times \mathbb{K}$ of the form

$$
y(t, z) \in \mathbb{Q}(t)[z]^{m},
$$

and a scalar function $z(t) \in \mathrm{QA}(\mathcal{U})$ such that

$$
\mathbf{z}=z(\mathbf{t}), \mathbf{y}=y(\mathbf{t}, \mathbf{z}),
$$

and for every $f(y) \in \mathbb{Q}[y]^{p}$, where $y=\left(y_{1}, \ldots, y_{m}\right)$ is a vector of indeterminates, such that $f(\mathbf{y})=0$, we have

$$
f(y(t, z))=0 .
$$

Moreover, the function $z(t)$ is algebraic of degree $d$ over $\mathbb{Q}(t)$.

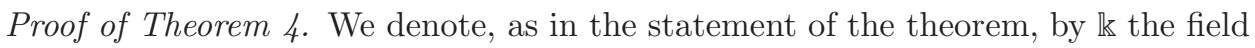
extension of $\mathbb{Q}$ generated by the components of $\mathbf{y}$, so $\mathbb{k} \subset \mathbb{K}$. Since there are finitely many such coefficients, $\mathbb{k}$ is a finitely generated field extension of $\mathbb{Q}$. Let $\mathbf{t}_{1}, \ldots$, $\mathbf{t}_{r}$ be a transcendence basis of $\mathbb{k}$ over $\mathbb{Q}$ (with $r \geq 1$ because $\mathbf{y} \notin \overline{\mathbb{Q}}^{m}$ ) and set 
$\mathbb{L}:=\mathbb{Q}\left(\mathbf{t}_{1}, \ldots, \mathbf{t}_{r}\right)$. By the primitive element theorem, there exists an element $\mathbf{z} \in \mathbb{k}$ finite over $\mathbb{L}$ and such that $\mathbb{L}(\mathbf{z})=\mathbb{k}$.

For every $i=1, \ldots, m$ we can write

$$
\mathbf{y}_{i}=\sum_{k=0}^{d-1} \frac{p_{i, k}\left(\mathbf{t}_{1}, \ldots, \mathbf{t}_{r}\right)}{q_{i, k}\left(\mathbf{t}_{1}, \ldots, \mathbf{t}_{r}\right)} \mathbf{z}^{k}
$$

where $d$ is the degree of $\mathbf{z}$ over $\mathbb{L}, p_{i, k}(t), q_{i, k}(t) \in \mathbb{Q}[t]$ and $q_{i, k}\left(\mathbf{t}_{1}, \ldots, \mathbf{t}_{r}\right) \neq 0$. By multiplying the $p_{i, k}$ by some well-chosen polynomials we may assume that all the $q_{i, k}(t)$ are equal, let us say to $q(t)$.

Let $P(t, z) \in \mathbb{Q}(t)[z]$ be the monic polynomial of minimal degree in $z$ such that

$$
P\left(\mathbf{t}_{1}, \ldots, \mathbf{t}_{r}, \mathbf{z}\right)=0 .
$$

Let $D \subset \mathbb{K}^{r}$ be the discriminant locus of $P(t, z)$ seen as a polynomial in $z$ (i.e. $D$ is the locus of points $a \in \mathbb{K}^{r}$ such that $a$ is a pole of one of the coefficients of $P$ or such that $P(a, z)$ has at least one multiple root). Since $P\left(\mathbf{t}_{1}, \ldots, \mathbf{t}_{r}, z\right)$ has no multiple roots in an algebraic closure of $\mathbb{L}$, the point $\mathbf{t}$ is not in $D$. Then there exist $\mathcal{U} \subset \mathbb{K}^{r} \backslash D$ a simply connected open neighborhood of $\mathbf{t}$ and analytic functions

$$
w_{i}: \mathcal{U} \longrightarrow \mathbb{K}, \quad i=1, \ldots, d
$$

such that

$$
P(t, z)=\prod_{i=1}^{d}\left(z-w_{i}(t)\right)
$$

and $w_{1}\left(\mathbf{t}_{1}, \ldots, \mathbf{t}_{r}\right)=\mathbf{z}$.

Moreover the $t \longmapsto w_{i}(t)$ are algebraic functions over $\mathbb{Q}[t]$. Let us set $\mathbb{Q}_{\mathbb{K}}=\mathbb{Q}$ when $\mathbb{K}=\mathbb{R}$ and $\mathbb{Q}_{\mathbb{K}}=\mathbb{Q}+i \mathbb{Q}$ when $\mathbb{K}=\mathbb{C}$. Then $w_{1} \in \mathrm{QA}(\mathcal{U})$ and the Taylor series of $w_{1}$ at a point of $\mathcal{U} \cap \mathbb{Q}_{\mathbb{K}}^{r}$ is an algebraic power series whose coefficients belong to a finite field extension of $\mathbb{Q}$.

Since the polynomial $q$ is not vanishing at $\mathbf{t}$ the function

$$
t \in \mathbb{K}^{r} \backslash\{q=0\} \longmapsto \frac{1}{q(t)}
$$

is a Nash function whose Taylor series at a point of $\mathbb{Q}_{\mathbb{K}}^{r} \backslash\{q=0\}$ is an algebraic power series with rational coefficients.

Let $b:=\left(b_{1}, \ldots, b_{r}\right) \in \mathbb{Q}_{\mathbb{K}}^{r} \cap \mathcal{U} \backslash\{q=0\}$. Let us denote by $\varphi_{1}(t)$ the Taylor series of $w_{1}$. Then let $\varphi_{2}(t) \in \mathbb{Q}\langle t\rangle$ denote the Taylor series of $t \longmapsto \frac{1}{q(t)}$ at $b$. For simplicity we can make a translation and assume that $b$ is the origin of $\mathbb{K}^{r}$.

For every $i=1, \ldots, m$ let us define

$$
y_{i}\left(t_{1}, \ldots, t_{r}, z, v\right):=v \sum_{k=0}^{d-1} p_{i, k}\left(t_{1}, \ldots, t_{r}\right) z^{k}
$$

and

$$
y(t, z, v):=\left(y_{1}(t, z, v), \ldots, y_{m}(t, z, v)\right) .
$$

Let $f(y) \in \mathbb{Q}[y]^{p}$ such that $f(\mathbf{y})=0$. We have that

$$
f\left(y\left(\mathbf{t}_{1}, \ldots, \mathbf{t}_{r}, \mathbf{z}, \frac{1}{q(\mathbf{t})}\right)\right)=0
$$


or equivalently

$$
f\left(y\left(\mathbf{t}_{1}, \ldots, \mathbf{t}_{r}, \varphi_{1}\left(\mathbf{t}_{1}, \ldots, \mathbf{t}_{r}\right), \varphi_{2}\left(\mathbf{t}_{1}, \ldots, \mathbf{t}_{r}\right)\right)\right)=0 .
$$

But the function

$$
t \longmapsto f\left(y\left(t_{1}, \ldots, t_{r}, \varphi_{1}\left(t_{1}, \ldots, t_{r}\right), \varphi_{2}\left(t_{1}, \ldots, t_{r}\right)\right)\right)
$$

is an algebraic function over $\mathbb{Q}[t]$ and $\mathbf{t}_{1}, \ldots, \mathbf{t}_{r}$ are algebraically independent over $\mathbb{Q}$. Thus we have that

$$
f\left(y\left(t_{1}, \ldots, t_{r}, \varphi_{1}\left(t_{1}, \ldots, t_{r}\right), \varphi_{2}\left(t_{1}, \ldots, t_{r}\right)\right)\right)=0 .
$$

Indeed, write $f=\left(f_{1}, \ldots, f_{p}\right)$ and for each $i=1, \ldots, m$ consider the complex valued function

$$
\Phi_{i}(t)=f_{i}\left(y\left(t_{1}, \ldots, t_{r}, \varphi_{1}\left(t_{1}, \ldots, t_{r}\right), \varphi_{2}\left(t_{1}, \ldots, t_{r}\right)\right)\right) .
$$

Note that $\Phi_{i} \in \mathrm{QA}(\mathcal{U})$ because so is $w_{1}$. Let $P_{1, i} \in \mathbb{Q}\left[t_{1}, \ldots, t_{r}, z\right]$ be a polynomial of minimal degree such that $P_{1, i}\left(t, \Phi_{i}(t)\right)=0$. Note that $P_{1, i}$ is irreducible. Write

$$
P_{1, i}(t, z)=\sum_{k=0}^{\operatorname{deg} P_{1, i}} a_{i, k}(t) z^{k} .
$$

Then $a_{i, 0}(\mathbf{t})=0$ since $\Phi_{i}(\mathbf{t})=0$. Therefore $a_{i, 0}(t)=0$ because $\mathbf{t}_{1}, \ldots, \mathbf{t}_{r}$ are algebraically independent over $\mathbb{Q}$. Hence $P_{1, i}=P_{2, i} z$ for some polynomial $P_{2, i}$, and $P_{2, i}$ is a unit since $P_{1, i}$ is irreducible. Therefore $\Phi_{i}(t)$ vanishes identically on $\mathcal{U}$.

This proves the theorem by defining

$$
y(t, z)=y\left(t, z, \varphi_{2}(t)\right)
$$

and $z(t)=w_{1}(t)$. Indeed the coefficients of the components of $y\left(t, z, \varphi_{2}(t)\right)$ seen as polynomials in $z$ are rational power series by (2.1). Moreover $\varphi_{1}(t)$ belongs to a finite field extension of $\mathbb{Q}(t)$ of degree $d$ since $P(t, z)$ is irreducible.

The following lemma will be used in the proof of the main theorem:

Lemma 5. Let $\varphi(t) \in \mathbb{C}\langle t\rangle$ be a power series algebraic over $\mathbb{Q}[t]$ and $P(t, \Phi) \in$ $\mathbb{Q}[t, \Phi]$ be a nonzero polynomial of degree $d$ in $\Phi$ such that $P(t, \varphi(t))=0$. Let $\mathbf{t} \in \mathbb{Q}^{r}$ be such that $\mathbf{t}$ is not in the vanishing locus of the coefficients of $P(t, \Phi)$ seen as a polynomial in $\Phi$, and such that $\mathbf{t}$ belongs to the domain of convergence of $\varphi(t)$. Then $\varphi(\mathbf{t})$ is an algebraic number of degree $\leq d$ over $\mathbb{Q}$.

Proof. The proof is straightforward: just replace $t$ by $\mathbf{t}$ in the relation $P(t, \varphi(t))=$ 0.

\section{ZARISKI EQUISINGULARITY}

Zariski equisingularity of families of singular varieties was introduced by Zariski in Za71] (originally it was called the algebro-geometric equisingularity). Answering a question of Zariski, Varchenko showed Va72, Va73, Va75] that Zariski equisingular families are locally topologically trivial. In the papers Va73, Va75] Varchenko considers the families of local singularities while the paper Va72] deals with the families of affine or projective algebraic varieties. A new method of proof of topological triviality, giving much stronger statements, was given recently in PP17. The case of families of algebraic varieties was considered in sections 5 and 9 of PP17. The version presented below follows from the proof of the main theorem, 
Theorem 3.3, of [PP17, see also Theorems 3.1 and 4.1 of Va72] in the complex case and Theorems 6.1 and 6.3 Va72 in the real case, and Proposition 5.2 and Theorem 9.2 of [PP17] where the algebraic global case is treated.

Theorem 6. Va72, PP17 Let $\mathcal{V}$ be an open connected neighborhood of $\mathbf{t}$ in $\mathbb{K}^{r}$ and let $\mathcal{O}_{\mathcal{V}}$ denote the ring of $\mathbb{K}$-analytic functions on $\mathcal{V}$. Let $t=\left(t_{1}, \ldots, t_{r}\right)$ denote the variables in $\mathcal{V}$ and let $x=\left(x_{1}, \ldots, x_{n}\right)$ be a set of variables in $\mathbb{K}^{n}$. Suppose that for $i=k_{0}, \ldots, n$, there are given

$$
F_{i}\left(t, x^{i}\right)=x_{i}^{d_{i}}+\sum_{j=1}^{d_{i}} a_{i-1, j}\left(t, x^{i-1}\right) x_{i}^{d_{i}-j} \in \mathcal{O}_{\mathcal{V}}\left[x^{i}\right],
$$

with $d_{i}>0$, such that

(i) for every $i>k_{0}$, the first non identically equal to zero generalized discriminant of $F_{i}\left(t, x^{i-1}, x_{i}\right)$ equals $F_{i-1}\left(t, x^{i-1}\right)$ up to a multiplication by a nowhere vanishing function of $\mathcal{O}_{\mathcal{V}}$.

(ii) the first non identically zero generalized discriminant of $F_{k_{0}}$ is independent of $x$ and does not vanish on $\mathcal{V}$.

Let us set for every $\mathbf{q} \in \mathcal{V}, V_{\mathbf{q}}=\left\{(\mathbf{q}, \mathbf{x}) \in \mathcal{V} \times \mathbb{K}^{n} \mid F_{n}(\mathbf{q}, \mathbf{x})=0\right\}$.

Then for every $\mathbf{q} \in \mathcal{V}$ there is a homeomorphism

$$
h_{\mathbf{q}}:\{\mathbf{t}\} \times \mathbb{K}^{n} \rightarrow\{\mathbf{q}\} \times \mathbb{K}^{n}
$$

such that $h_{\mathbf{q}}\left(V_{\mathbf{t}}\right)=V_{q}$.

Moreover if $F_{n}=G_{1} \cdots G_{s}$ then for every $j=1, \ldots, s$

$$
h_{\mathbf{q}}\left(G_{j}^{-1}(0) \cap\left(\{\mathbf{t}\} \times \mathbb{K}^{n}\right)\right)=G_{j}^{-1}(q) \cap\left(\{0\} \times \mathbb{K}^{n}\right) .
$$

For the notion of generalized discriminants see subsection 6.3

Remark 7. The homeomorphism $h_{\mathbf{q}}$ of Theorem [6 can be written $h_{\mathbf{q}}(\mathbf{t}, x)=$ $\left(\mathbf{q}, \Psi_{\mathbf{q}}(x)\right)$, i.e., as a family of homeomorphisms $\Psi_{\mathbf{q}}: \mathbb{K}^{n} \rightarrow \mathbb{K}^{n}$. If $F_{i}$ are homogeneous polynomials in $x$, then the homeomorphisms $\Psi_{\mathbf{q}}$ satisfy, by construction,

$$
\forall \lambda \in \mathbb{K}^{*}, \forall x \in \mathbb{K}^{n} \quad \Psi_{\mathbf{q}}(\lambda x)=\lambda \Psi_{\mathbf{q}}(x)
$$

Hence if we define $\mathbb{P}\left(V_{\mathbf{q}}\right)=\left\{(\mathbf{q}, \mathbf{x}) \in \mathcal{V} \times \mathbb{P}_{\mathbb{K}}^{n} \mid F_{n}(\mathbf{q}, \mathbf{x})=0\right\}$, the homeomorphism $h_{\mathbf{q}}$ induces an homeomorphism between $\mathbb{P}\left(V_{\mathbf{t}}\right)$ and $\mathbb{P}\left(V_{\mathbf{q}}\right)$.

Remark 8. By construction of [Va72, Va73, Va75] and [PP17, the homeomorphisms $h_{\mathbf{q}}$ can be obtained by a local topological trivialization. That is there is a neighborhood $\mathcal{W}$ of $\mathbf{t}$ in $\mathbb{K}^{r}$ and a homeomorphism

$$
\Phi: \mathcal{W} \times \mathbb{K}^{n} \rightarrow \mathcal{W} \times \mathbb{K}^{n}
$$

such that $\Phi(\mathbf{q}, x)=\left(\mathbf{q}, h_{\mathbf{q}}(x)\right)$. Moreover, as follows from PP17, we may require that:

(1) The homeomorphism $\Phi$ is subanalytic. In the algebraic case, that is in the case considered in this paper, we replace in the assumptions $\mathcal{O}_{\mathcal{V}}$ by the ring of $\mathbb{K}$-valued Nash functions on $\mathcal{V}$. Then $\Phi$ can be chosen semialgebraic.

(2) $\Phi$ is arc-wise analytic, see Definition 1.2 of [PP17]. In particular each $h_{\mathbf{q}}$ is arc-analytic. It means that for every real analytic arc $\gamma(s):(-1,1) \rightarrow \mathbb{K}^{n}$, $h_{\mathbf{q}} \circ \gamma$ is real analytic and the same property holds for $h_{\mathbf{q}}^{-1}$. 


\section{Generic linear changes of Coordinates}

Let $f \in \mathbb{K}[x]$ be a polynomial of degree $d$ and let $\mathbb{k}$ be a field extension of $\mathbb{Q}$ containing the coefficients of $f$. We denote by $\bar{f}$ the homogeneous part of degree $d$ of $f$. The polynomial

$$
\bar{f}\left(x_{1}, \ldots, x_{n-1}, 1\right) \not \equiv 0
$$

otherwise $\bar{f}$ would be divisible by $x_{n}-1$ which is impossible since $\bar{f}$ is a homogeneous polynomial. So there exists

$$
\left(\mu_{1}, \ldots, \mu_{n-1}\right) \in \mathbb{Q}^{n-1}
$$

such that $c:=\bar{f}\left(\mu_{1}, \ldots, \mu_{n-1}, 1\right) \neq 0$. Then let us denote by $\varphi_{\mu}$ the linear change of coordinates defined by

$$
\begin{array}{ccc}
x_{i} & \longmapsto & x_{i}+\mu_{i} x_{n} \text { for } i<n \\
x_{n} & \longmapsto & x_{n} .
\end{array}
$$

We have that

$$
\varphi_{\mu}(\bar{f})=c x_{n}^{d}+h
$$

where $h$ is a homogeneous polynomial of degree $d$ belonging to the ideal generated by $x_{1}, \ldots, x_{n-1}$. So we have that

$$
\varphi_{\mu}(f)=c x_{n}^{d}+\sum_{j=1}^{d} a_{j}\left(x^{n-1}\right) x_{n}^{d-j}
$$

for some polynomials $a_{j}\left(x^{n-1}\right) \in \mathbb{k}\left[x^{n-1}\right]$.

Remark 9. If $g=g_{1} \ldots g_{s}$ is a product of polynomials of $\mathbb{k}[x]$, then the linear change of coordinates $\varphi_{\mu}$ defined above also satisfies

$$
\varphi_{\mu}\left(g_{i}\right)=c_{i} x_{n}^{d_{i}}+\sum_{j=1}^{d} a_{i, j}\left(x^{n-1}\right) x_{n}^{d-j}
$$

for some nonzero constants $c_{i} \in \mathbb{k}$.

Remark 10. For every $f \in \mathbb{k}[x]$ of degree $d$ where $\mathbb{k} \subset \mathbb{K}$ and $\mu \in \mathbb{Q}^{n-1}$, we have that

$$
\varphi_{-\mu} \circ \varphi_{\mu}(f)=f \text {. }
$$

Let us define the support of $f=\sum_{\alpha \in \mathbb{N}^{n}} f_{\alpha} x^{\alpha}$ as

$$
\operatorname{Supp}(f):=\left\{\alpha \in \mathbb{N}^{n} \mid f_{\alpha} \neq 0\right\} .
$$

Let us assume that

$$
\varphi_{\mu}(f)=\sum f_{\alpha}^{\prime} x^{\alpha}
$$

for some polynomials $f_{\alpha}^{\prime} \in \mathbb{k}$. Then the coefficient $f_{\alpha}$ has the form

$$
f_{\alpha}=P_{\alpha}\left(\mu_{1}, \ldots, \mu_{n-1}, f_{\beta}^{\prime}\right)
$$

for some polynomial $P_{\alpha} \in \mathbb{Z}\left[\mu, f_{\beta}^{\prime}\right]$ depending only on the $f_{\beta}^{\prime}$ with $|\beta|=|\alpha|$. 


\section{MAIN THEOREM}

We can state now our main result:

Theorem 11. Let $V \subset \mathbb{K}^{n}$ (resp. $V \subset \mathbb{P}_{\mathbb{K}}^{n}$ ) be an affine (resp. projective) algebraic set, where $\mathbb{K}=\mathbb{R}$ or $\mathbb{C}$. Then there exist an affine (resp. projective) algebraic set $W \subset \mathbb{K}^{n}$ (resp. $W \subset \mathbb{P}_{\mathbb{K}}^{n}$ ) and a homeomorphism $h: \mathbb{K}^{n} \longrightarrow \mathbb{K}^{n}$ (resp. $\left.h: \mathbb{P}_{\mathbb{K}}^{n} \longrightarrow \mathbb{P}_{\mathbb{K}}^{n}\right)$ such that:

(i) The homeomorphism maps $V$ onto $W$,

(ii) $W$ is defined by polynomial equations with coefficients in $\overline{\mathbb{Q}} \cap \mathbb{K}$,

(iii) The variety $W$ is obtained from $V$ by a Zariski equisingular deformation. In particular the homeomorphism $h$ can be chosen semialgebraic and arcanalytic,

(iv) Let $g_{1}, \ldots, g_{s}$ be the generators of the ideal defining $V$. Let us fix $\varepsilon>$ 0. Then $W$ can be chosen so that the ideal defining it is generated by polynomials $g_{1}^{\prime}, \ldots, g_{s}^{\prime} \in \overline{\mathbb{Q}}[x]$ such that if we write

$$
g_{i}=\sum_{\alpha \in \mathbb{N}^{n}} g_{i, \alpha} x^{\alpha} \text { and } g_{i}^{\prime}=\sum_{\alpha \in \mathbb{N}^{n}} g_{i, \alpha}^{\prime} x^{\alpha}
$$

then for every $i$ and $\alpha$ we have that:

$$
\left|g_{i, \alpha}-g_{i, \alpha}^{\prime}\right|<\varepsilon \text {. }
$$

(v) Every polynomial relationship with rational coefficients between the $g_{i, \alpha}$ will also be satisfied by the $g_{i, \alpha}^{\prime}$.

Remark 12. Arc-analytic semialgebraic maps were introduced first in Ku88. By BM90 they coincide with the category of blow-Nash maps (i.e. blow-analytic and semialgebraic maps), and thus as blow-analytic homeomorphisms they appeared already in the classification of real singularities in Kuo85. Arc-analytic semialgebraic maps and homeomorphisms are often used in real algebraic geometry. They share many properties of regular morphisms but are more flexible. For instance, arc-analytic semialgebraic homeomorphisms preserve the weight fitration and the virtual Poincarè polynomial MP2011, but the equivalence relation defined by such homeomorphisms does not have continuous moduli PP17. For more, the interested reader may consult the survey [KP08] and the Kucharz-Kurdyka talk on 2018 ICM $\mathrm{KK} 18$.

Remark 13. We will see in the proof the following: let $\mathbb{k}$ denote the field extension of $\mathbb{Q}$ generated by the coefficients of the $g_{i}$ and assume that $\mathbb{k} \neq \overline{\mathbb{Q}}$ (otherwise there is nothing to prove). By the primitive element theorem, $\mathbb{k}$ is a simple extension of a purely transcendental extension $\mathbb{L}$ of $\mathbb{Q}$, i.e. $\mathbb{Q} \longrightarrow \mathbb{L}$ is purely transcendental and $\mathbb{L} \longrightarrow \mathbb{k}$ is a simple extension of degree $d$. Then the coefficients of the $g_{i}^{\prime}$ belong to a finite extension of $\mathbb{Q}$ of degree $\leq d$.

In particular if $\mathbb{Q} \longrightarrow \mathbb{k}$ is purely transcendental then $W$ is defined over $\mathbb{Q}$.

Remark 14. In fact (v) implies that

$$
\forall i, \alpha \quad g_{i, \alpha}=0 \Longrightarrow g_{i, \alpha}^{\prime}=0 .
$$

If $\varepsilon$ is chosen small enough we may even assume that

$$
\forall i, \alpha \quad g_{i, \alpha}=0 \Longleftrightarrow g_{i, \alpha}^{\prime}=0 .
$$


Proof of Theorem 11. Let us consider a finite number of polynomials $g_{1}, \ldots, g_{s} \in$ $\mathbb{K}[x]$ generating the ideal defining $V$ and let us denote by $g_{i, \alpha}$ their coefficients as written in the theorem. After a linear change of coordinates $\varphi_{\mu}$ with $\mu \in \mathbb{Q}^{n-1}$ as in Section 4 we can assume that

$$
\varphi_{\mu}\left(g_{r}\right)=c_{r} x_{n}^{p_{r}}+\sum_{j=1}^{p_{r}} b_{n-1, r, j}\left(x^{n-1}\right) x_{n}^{p_{r}-j}=\sum_{\beta \in \mathbb{N}^{n}} a_{n, r, \beta} x^{\beta} \quad \forall r=1, \ldots, s .
$$

Moreover by multiplying each $\varphi_{\mu}\left(g_{r}\right)$ by $1 / c_{r}$ we can assume that $c_{r}=1$ for every $r$. We denote by $f_{n}$ the product of the $\varphi_{\mu}\left(g_{r}\right)$ and by $a_{n}$ the vectors of coefficients $a_{n, r, \beta}$. The entries of $a_{n}$ are polynomial functions in the $g_{i, \alpha}$ with rational coefficients, let us say

$$
a_{n}=A_{n}\left(g_{i, \alpha}\right)
$$

for some $A_{n}=\left(A_{n, r, \beta}\right)_{r, \beta} \in \mathbb{Q}\left(u_{i, \alpha}\right)^{N_{n}}$ for some integer $N_{n}>0$ and some new indeterminates $u_{i, \alpha}$.

Let $l_{n}$ be the smallest integer such that

$$
\Delta_{n, l_{n}}\left(a_{n}\right) \not \equiv 0
$$

where $\Delta_{n, l}$ denotes the $l$-th generalized discriminant of $f_{n}$. In particular we have that

$$
\Delta_{n, l}\left(a_{n}\right) \equiv 0 \quad \forall l<l_{n} .
$$

After a linear change of coordinates $x^{n-1}$ with coefficients in $\mathbb{Q}$ we can write $\Delta_{n, l_{n}}\left(a_{n}\right)=e_{n-1} f_{n-1}$ with

$$
f_{n-1}=\sum_{\beta \in \mathbb{N}^{n}} a_{n-1, \beta} x^{\beta}=x_{n-1}^{d_{n-1}}+\sum_{j=1}^{d_{n-1}} b_{n-2, j}\left(x^{n-2}\right) x_{n-1}^{d_{n-1}-j}
$$

for some constants $e_{n-1}, a_{n-1, \beta} \in \mathbb{k}$, with $e_{n-1} \neq 0$, and some polynomials $b_{n-2, j} \in$ $\mathbb{k}\left[x^{n-2}\right]$. We denote by $a_{n-1}$ the vector of coefficients $a_{n-1, \beta}$. Let $l_{n-1}$ be the smallest integer such that

$$
\Delta_{n-1, l_{n-1}}\left(a_{n-1}\right) \not \equiv 0
$$

where $\Delta_{n-1, l}$ denotes the $l$-th generalized discriminant of $f_{n-1}$.

We repeat this construction and define a sequence of polynomials $f_{j}\left(x^{j}\right), j=$ $k_{0}, \ldots, n-1$ for some $k_{0}$, such that

$$
\Delta_{j+1, l_{j+1}}\left(a_{j+1}\right)=e_{j}\left(x_{j}^{d_{j}}+\sum_{k=1}^{d_{j}} b_{j-1, k}\left(x^{j-1}\right) x_{j}^{d_{j}-k}\right)=e_{j}\left(\sum_{\beta \in \mathbb{N}^{n}} a_{j, \beta} x^{\beta}\right)=e_{j} f_{j}
$$

is the first non identically zero generalized discriminant of $f_{j+1}$, where $a_{j}$ denotes the vectors of coordinates $a_{j, \beta}$ in $\mathbb{k}^{N_{j}}$, i.e. we have that:

$$
\Delta_{j+1, l_{j+1}}\left(a_{j+1}\right)=e_{j}\left(\sum_{\beta \in \mathbb{N}^{n}} a_{j, \beta} x^{\beta}\right)=e_{j} f_{j}
$$

and

$$
\Delta_{j+1, l}\left(a_{j+1}\right) \equiv 0 \quad \forall l<l_{j+1},
$$

until we get $f_{k_{0}}=1$ for some $k_{0} \geq 1$. 
By (5.2) we see that the entries of $a_{j}$ and $e_{j}$ are rational functions in the entries of $a_{j+1}$ for every $j<n$. Thus by (5.1) we see that the entries of the $a_{k}$ and the $e_{j}$ are rational functions in the $g_{i, \alpha}$ with rational coefficients, let us say

$$
a_{k}=A_{k}\left(g_{i, \alpha}\right), \quad e_{j}=E_{j}\left(g_{i, \alpha}\right)
$$

for some $A_{k} \in \mathbb{Q}\left(u_{i, \alpha}\right)^{N_{k}}$ and $E_{j} \in \mathbb{Q}\left(u_{i, \alpha}\right)$ for every $k$ and $j$, where the $u_{i, \alpha}$ are new indeterminates for every $i$ and $\alpha$.

By Theorem 4 there exist a new set of indeterminates $t=\left(t_{1}, \ldots, t_{r}\right)$, an open domain $\mathcal{U} \subset \mathbb{K}^{r},\left(\mathbf{t}_{1}, \ldots, \mathbf{t}_{r}\right) \in \mathcal{U}$, polynomials $g_{i, \alpha}(t, z) \in \mathbb{Q}(t)[z]$, for every $i$ and $\alpha$, and $z(t) \in \mathrm{QA}(\mathcal{U})$ such that $g_{i, \alpha}(\mathbf{t}, z(\mathbf{t}))=g_{i, \alpha}$ for every $i$ and $\alpha$.

Since the $a_{l}$ and the $e_{j}$ are rational functions with rational coefficients in the $g_{i, \alpha}$, the system of equations (5.3) is equivalent to a system of polynomial equations with rational coefficients

$$
f\left(g_{i, \alpha}\right)=0 .
$$

By Theorem 4 the functions $g_{i, \alpha}(t, z(t))$ are solutions of this system of equations.

Because $e_{j}\left(g_{i, \alpha}\right)=e_{j}\left(g_{i, \alpha}(\mathbf{t}, z(\mathbf{t}))\right) \neq 0$ for every $j$, we have that none of the $e_{j}\left(g_{i, \alpha}(t, z(t))\right)$ vanishes in a small open ball $\mathcal{V} \subset \mathcal{U}$ centered at $\mathbf{t}$.

In particular we have that

$$
e_{j}\left(g_{i, \alpha}(t, z(t))\right) \neq 0 \quad \forall j, \forall t \in \mathcal{V} .
$$

For $t \in \mathcal{V}$ and $r=1, \ldots, s$, we define

$$
\begin{aligned}
g_{r}^{\prime}(t, x) & :=\sum_{\alpha \in \mathbb{N}^{n}} g_{i, \alpha}(t, z(t)) x^{\alpha}, \\
F_{n}(t, x) & =\prod_{r=1}^{s} G_{r}(t, x), \text { where } \\
G_{r}(t, x) & =\sum_{\alpha \in \mathbb{N}^{n}} A_{n, r, \alpha}\left(g_{i, \alpha}(t, z(t))\right) x^{\alpha}, r=1, \ldots, s,
\end{aligned}
$$

and for $j=k_{0}, \ldots, n-1$

$$
F_{j}(t, x)=\sum_{\alpha \in \mathbb{N}^{n}} A_{j, \alpha}\left(g_{i, \alpha}(t, z(t))\right) x^{\alpha} .
$$

In particular we have that $G_{r}(\mathbf{t}, x)=\varphi_{\mu}\left(g_{r}(x)\right)$ for every $r$.

Let us denote by $\mathcal{O}_{\mathcal{V}}$ the ring of $\mathbb{K}$-analytic functions on $\mathcal{V}$. In particular we have that $F_{j}(t, x)$ is a polynomial of $\mathcal{O}_{\mathcal{V}}\left[x^{i}\right]$, of degree $d_{j}$ in $x_{j}$, such that the coefficient of $x_{j}^{d_{j}}$ is $e_{j}\left(g_{i, \alpha}(t, z(t))\right)$ and whose discriminant (seen as a polynomial in $x_{j}$ ) is equal to $e_{j-1}\left(g_{i, \alpha}(t, z(t))\right) F_{j-1}(t, x) \in \mathcal{O}_{\mathcal{V}}\left[x^{j-1}\right]$ for every $j$ by (5.4).

Thus the family $\left(F_{j}\right)_{j}$ satisfies the hypothesis of Theorem 6 by (5.5). Hence the algebraic hypersurfaces $X_{0}:=\left\{F_{n}(0, x)=0\right\}$ and $X_{1}:=\left\{F_{n}(1, x)\right\}$ are homeomorphic. Moreover the homeomorphism between them maps every component of $X_{0}$ defined by $G_{r}(0, x)=0$ onto the component of $X_{1}$ defined by $G_{r}(1, x)=0$. This proves that the algebraic variety defined by $\left\{\varphi_{\mu}\left(g_{1}\right)=\cdots=\varphi_{\mu}\left(g_{s}\right)=0\right\}$ is homeomorphic to the algebraic variety $\left\{G_{1}(0, x)=\cdots=G_{s}(0, x)=0\right\}$ which is defined by polynomial equations over $\overline{\mathbb{Q}}$. Thus $V$ is homeomorphic to $W:=\left\{g_{1}^{\prime}(x)=\cdots=\right.$ $\left.g_{s}^{\prime}(x)=0\right\}$. 
VARIETIES ARE HOMEOMORPHIC TO VARIETIES DEFINED OVER NUMBER FIELDS 11

Moreover since $\overline{\mathbb{Q}} \cap \mathbb{K}$ is dense in $\mathbb{K}$, we can choose $\mathbf{q} \in \overline{\mathbb{Q}}$ as close as we want to $\mathbf{t}$. In particular by choosing $\mathbf{q}$ close enough to $\mathbf{t}$ we may assume that (iv) in Theorem 11 is satisfied, since $\gamma, t \mapsto z(t)$ and the $g_{i, \alpha}$ are continuous functions.

Finally we have that $z(\mathbf{q})$ is algebraic of degree $\leq d$ over $\mathbb{Q}$ by Lemma $\$$ (see Remark 13).

Thus Theorem 11 is proven in the affine case. The projective is proven is the same way by Remark 7

We can remark that Theorem 11(v) implies that several algebraic invariants are preserved by the homeomorphism $h$. For instance we have the following corollary:

Corollary 15. For $\varepsilon>0$ small enough the Hilbert-Samuel function of $W$ is the same as that of $V$.

Proof of Corollary [15, Let $h_{1}, \ldots, h_{m}$ be a Gröbner basis of the ideal $I$ of $\mathbb{K}[x]$ generated by the $g_{i}$ with respect to a given monomial order $\preceq$. Let us recall that for $f=\sum_{\alpha \in \mathbb{N}^{n}} f_{\alpha} x^{\alpha} \in \mathbb{K}[x], f \neq 0$, we denote the leading term of $f$ by $\operatorname{LT}(f)=f_{\alpha_{0}} x^{\alpha_{0}}$ where $\alpha_{0}$ is the largest nonzero exponent of the support of $f$ with respect to the monomial order:

$$
\operatorname{Supp}(f)=\left\{\alpha \in \mathbb{N}^{n} \mid f_{\alpha} \neq 0\right\}
$$

A Gröbner basis of $I$ is computed by considering S-polynomials and divisions (see CLO07, for more details):

- the S-polynomial of two nonzero polynomials $f$ and $g$ is defined as follows: set $\mathrm{LT}(f)=a x^{\alpha}$ and $\operatorname{LT}(g)=b x^{\beta}$ with $a, b \in \mathbb{K}^{*}$, and let $x^{\delta}$ be the least common multiple of $x^{\alpha}$ and $x^{\beta}$. Then the S-polynomial of $f$ and $g$ is

$$
S(f, g):=\frac{x^{\delta-\alpha}}{a} f-\frac{x^{\delta-\beta}}{b} g .
$$

- the division of a polynomial $f$ by polynomials $f_{1}, \ldots, f_{l}$ is defined inductively as follows: firstly after some renumbering one assume that $\operatorname{LT}\left(f_{1}\right) \preceq \operatorname{LT}\left(f_{2}\right) \preceq \cdots \preceq$ $\mathrm{LT}\left(f_{l}\right)$. Then we consider the smallest integer $i$ such that LT $(f)$ is divisible by $\mathrm{LT}\left(f_{i}\right)$. If such a $i$ exists one sets $q_{i}^{(1)}=\frac{\mathrm{LT}(f)}{\mathrm{LT}\left(f_{i}\right)}$ and $q_{j}^{(1)}=0$ for $j \neq i$ and $r^{(1)}=0$. Otherwise one sets $q_{j}^{(1)}=0$ for every $j$ and $r^{(1)}=\mathrm{LT}(f)$. We repeat this process by replacing $f$ by $f-\sum_{j=1}^{l} q_{j}^{(1)} f_{j}-r^{(1)}$. After a finite number of steps we obtain a decomposition

$$
f=\sum_{j=1}^{l} q_{j} f_{j}+r
$$

where none of the elements of $\operatorname{Supp}(r)$ is divisible by any $\operatorname{LT}\left(f_{i}\right)$. The polynomial $r$ is called the remainder of the division of $f$ by the $f_{i}$.

Thus we can make the following remark: every remainder of the division of some $S$-polynomial $S\left(g_{i}, g_{j}\right)$ by $g_{1}, \ldots, g_{s}$ is a polynomial whose coefficients are rational functions on the coefficients of the $g_{k}$.

The Buchberger's Algorithm is as follows: we begin with $g_{1}, \ldots, g_{s}$ the generators of $I$ and we compute all the S-polynomials of every pair of polynomials among the $g_{i}$. Then we consider the remainders of the divisions of these S-polynomials by the $g_{i}$. If some remainders are nonzero we add them to the family $\left\{g_{1}, \ldots, g_{s}\right\}$. Then 
we repeat the same process that stops after a finite number of setps.

By the previous remark if $h$ denotes the remainder of the division of a S-polynomial $S\left(g_{i}, g_{j}\right)$ by the $g_{k}$ then we have a relation of the form

$$
S\left(g_{i}, g_{j}\right)=\sum_{l=1}^{s} b_{l} g_{l}+h
$$

and the $b_{l}$ belong to the field extension of $\mathbb{Q}$ generated by the coefficients of the $g_{l}$. Let $h_{\beta}$ denote the coefficient of $x^{\beta}$ in $h$. Then for those $h_{\beta}$ that are nonzero, Equation (5.6) provides an expression of them as rational functions in the $g_{i, \alpha}$, let us say

$$
\forall \beta \in \operatorname{Supp}(h) \quad h_{\beta}=H_{\beta}\left(g_{i, \alpha}\right) .
$$

For those $h_{\beta}$ equal to zero Equation (5.6) provides a polynomial relation with rational coefficients between the $g_{i, \alpha}$ :

$$
\forall \beta \notin \operatorname{Supp}(h) \quad H_{\beta}\left(g_{i, \alpha}\right)=0 .
$$

And Equation (5.6) is equivalent to the systems of equations (5.7) and (5.8). This remains true if we replace the $g_{i}$ by polynomials whose coefficients are rational functions in the $g_{i, \alpha}$ with rational coefficients. Thus the fact that $h_{1}, \ldots, h_{m}$ is a Gröbner basis obtained from the $g_{i}$ by Buchberger's Algorithm is equivalent to a system of equations

$$
Q_{k}\left(g_{i, \alpha}\right)=0 \text { for } k \in E
$$

where $E$ is a (not necessarily finite) set. Thus by Theorem 11(v) we see that these equations are satisfied by the coefficients of the $g_{i}^{\prime}$, hence the ideal defining $W$ has a Gröbner basis $h_{1}^{\prime}, \ldots, h_{m}^{\prime}$ obtained from the $g_{i}^{\prime}$ by doing exactly the same steps in Buchberger's Algorithm, and $\operatorname{Supp}\left(h_{i}^{\prime}\right) \subset \operatorname{Supp}\left(h_{i}\right)$ for every $i$.

Moreover the initial terms of the $h_{i}$ are rational functions in the $g_{i, \alpha}$ with rational coefficients, let us say $H_{i}\left(g_{i, \alpha}\right)$ for some rational functions $H_{i}$. By choosing $\varepsilon$ small enough we insure that

$$
H_{i}\left(g_{i, \alpha}^{\prime}\right) \neq 0 \quad \forall i .
$$

Thus the leading exponents of the $h_{i}^{\prime}$ are equal to those of the $h_{i}$. In particular the Hilbert-Samuel function of $W$ is the same as that of $V$, and (iv) in Theorem 11 is proven.

Remark 16. In fact we have proven that the ideal of leading terms of $I$ is the same as the ideal of leading terms of the ideal defining $W$. So we could have concluded by [GP08, Theorem 5.2.6] for instance.

\section{Complete Algorithm}

\subsection{Settings.}

\section{Input:}

(1) polynomials $g_{1}, \ldots, g_{s} \in \mathbb{K}[x]$ whose coefficients $g_{i, \alpha}$ belong to a finitely generated field extension $\mathbb{k}$ over $\mathbb{Q}$.

(2) a presentation

$$
\mathbb{k}=\mathbb{Q}\left(\mathbf{t}_{1}, \ldots, \mathbf{t}_{r}, \mathbf{z}\right)
$$

where the $\mathbf{t}_{i}$ are algebraically independent over $\mathbb{Q}$ and $\mathbf{z}$ is finite over $\mathbb{Q}\left(\mathbf{t}_{1}, \ldots, \mathbf{t}_{r}\right)$.

(3) the minimal polynomial $P(z)$ of $\mathbf{z}$ over $\mathbb{Q}\left(\mathbf{t}_{1}, \ldots, \mathbf{t}_{r}\right)$. 
(4) the $g_{i, \alpha}$ are given as rational functions in the $\mathbf{t}_{i}$ and $\mathbf{z}$, and the coefficients of $P(z)$ as rational functions in the $\mathbf{t}_{i}$.

(5) a positive real number $\varepsilon$.

Moreover we assume that the $\mathbf{t}_{i}$ and $\mathbf{z}$ are computable numbers Tu36]. Let us recall that a real computable number is a number $\mathbf{x} \in \mathbb{R}$ for which there is a Turing machine that computes a sequence $\left(\mathbf{q}_{n}\right)$ of rational numbers such that $\left|\mathbf{x}-\mathbf{q}_{n}\right|<\frac{1}{n}$ for every $n \geq 1$. A computable number is a complex number whose real and imaginary parts are real computable numbers. The set of computable numbers is an algebraically closed field Ri54. More precisely if $\mathbf{t}_{1}, \ldots, \mathbf{t}_{r}$ are computable numbers such that for every $i \in\{1, \ldots, r\}\left(\mathbf{q}_{i, n}\right)_{n}$ is a sequence of $\mathbb{Q}+i \mathbb{Q}$ computed by a Turing machine with $\left|\mathbf{t}_{i}-\mathbf{q}_{i, n}\right|<\frac{1}{n}$ for every $n$, and if $\mathbf{z} \in \mathbb{C}$ satisfies $P(\mathbf{t}, \mathbf{z})=0$ for some reduced polynomial $P(t, z) \in \mathbb{Q}[t, z]$, then one can effectively find a Turing machine that computes a sequence $\left(\mathbf{q}_{n}\right)_{n}$ of $\mathbb{Q}+i \mathbb{Q}$ such that $\left|\mathbf{z}-\mathbf{q}_{n}\right|<\frac{1}{n}$ for every integer $n$.

Output: polynomials $g_{1}^{\prime}, \ldots, g_{s}^{\prime} \in(\overline{\mathbb{Q}} \cap \mathbb{K})[x]$ with the properties:

(1) the pairs $\left(V\left(g_{i}\right), \mathbb{K}^{n}\right)$ and $\left(V\left(g_{i}^{\prime}\right), \mathbb{K}^{n}\right)$ are homeomorphic, and the homeomorphism is semialgebraic and arc-analytic.

(2) the coefficients $g_{i, \alpha}^{\prime}$ of $g_{i}^{\prime}$ satisfy the properties:

$$
\begin{gathered}
g_{i, \alpha}^{\prime} \neq 0 \Longleftrightarrow g_{i, \alpha} \neq 0 \\
\left|g_{i, \alpha}^{\prime}-g_{i, \alpha}\right|<\varepsilon \text { for every } i \text { and } \alpha
\end{gathered}
$$

and every polynomial relation with coefficients in $\mathbb{Q}$ satisfied by the $g_{i, \alpha}$ is also satisfied by the $g_{i, \alpha}^{\prime}$.

6.2. Algorithm: We present here the successive steps of the algorithm.

(1) We make a linear change of coordinates with coefficients in $\mathbb{Q}$, denoted by $\varphi_{\mu}$ with $\mu \in \mathbb{Q}^{n-1}$, such that each of the $g_{i}$ is a monic polynomial in $x_{n}$ of degree $\operatorname{deg}\left(g_{i}\right)$.

We denote by $f_{n}$ the product of the $g_{i}$ after this change of coordinates, and by $a_{n}$ the vector of the coefficients of the $g_{i}$ after this change of coordinates (seen as a polynomial in $x_{n}$ ).

(2) For every $j$ from $n$ to 1 we do the following: let us assume that $f_{j}$ is a polynomial in $x_{1}, \ldots, x_{j}$ having a nonzero monomial $e_{j} x_{j}^{\operatorname{deg}\left(f_{j}\right)}, e_{j} \in \mathbb{k}^{*}$. We denote by $a_{j}$ the vector of the coefficients of $f_{j}$ seen as a polynomial in $x_{j}$. We consider the generalized discriminants $\Delta_{j, l}$ of $f_{j}$ with respect to $x_{j}$, and we denote by $l_{j}$ the smallest integer such that

$$
\Delta_{j, l_{j}}\left(a_{j}\right) \neq 0 .
$$

These polynomials $\Delta_{j, l_{j}}\left(a_{j}\right)$ can be effectively computed (see 6.3).

We perform a linear change of coordinates (with coefficients in $\mathbb{Q}$ ) in $x_{1}, \ldots, x_{j-1}$ such that $\Delta_{j, l_{j}}\left(a_{j}\right)$ becomes a unit times a monic polynomial of degree $\operatorname{deg}\left(\Delta_{j, l_{j}}\left(a_{j}\right)\right)$ in $x_{j-1}$, and we denote by $f_{j-1}$ this new monic polynomial.

(3) We stop the process once we have that $f_{j-1}$ is a nonzero constant.

(4) We consider (5.2) as a system of polynomial equations that will allow us to 
compute the expression of the $e_{j}$ as rational functions in the $g_{i, \alpha}$.

(5) We denote by $P(t, z)$ the monic polynomial of $\mathbb{Q}(t)[z]$ such that $P(\mathbf{t}, z)=P(z)$ is the minimal polynomial of $\mathbf{z}$ over $\mathbb{Q}(\mathbf{t})$.

By replacing $\varepsilon$ by a smaller positive number we may assume that

$$
d\left(\mathbf{t}, \Delta_{P}\right)>2 \varepsilon
$$

where $\Delta_{P}$ denotes the discriminant locus of $P(t, z)$ seen as a polynomial in $z$. This discriminant is computed as $\operatorname{Res}_{z}(P, \partial P / \partial z)$. See [DGY96] or [BM95, Theorem C] for a practical way of choosing such a $\varepsilon$.

(6) Let $\mathbf{z}_{1}:=\mathbf{z}, \mathbf{z}_{2}, \ldots, \mathbf{z}_{d}$ be the distincts roots of $P(\mathbf{t}, z)$. These are computable numbers and so we can compute $\eta \in \mathbb{Q}>0$ such that all the differences between two $\mathbf{z}_{i}$ are strictly greater than $\eta$.

Let $z(t)$ be the root of $P(t, z)$ such that $z(\mathbf{t})=\mathbf{z}$. We can write

$$
w(t):=z(t+\mathbf{t})=\sum_{\alpha \in \mathbb{N}^{r}} \mathbf{z}_{\alpha} t^{\alpha}, \quad \mathbf{z}_{0}=\mathbf{z} .
$$

We write

$$
P(t, z)=p_{0}(t)+p_{1}(t) z+\cdots+p_{d-1}(t) z^{d-1}+z^{d}
$$

where the $p_{i}(t) \in \mathbb{K}(t)$. Set

$$
M:=1+\max _{0 \leq i \leq d-1} \max _{\mathbf{t}^{\prime} \in B(0,2 \varepsilon)}\left|p_{i}\left(\mathbf{t}^{\prime}\right)\right| .
$$

Then $\left|\mathbf{z}_{\alpha}\right| \leq \frac{M}{(2 \varepsilon)^{|\alpha|}}$ for every $\alpha$ (by the Cauchy bounds for the roots of a monic polynomial since $w(t)$ is convergent on $B(0,2 \varepsilon)$ by $(5))$. Let $W_{k}(t)$ be the homogeneous term of degree $k$ in the Taylor expansion of $w(t): W_{k}(t)=\sum_{|\alpha|=k} \mathbf{z}_{\alpha} t^{\alpha}$. Then

$$
\forall \mathbf{t}^{\prime} \in B(0, \varepsilon) \quad\left|W_{k}\left(\mathbf{t}^{\prime}\right)\right| \leq \frac{M}{2^{k}}\left(\begin{array}{c}
k+r-1 \\
r-1
\end{array}\right) .
$$

We have that

$$
\forall k \geq r \quad \frac{M}{2^{k}}\left(\begin{array}{c}
k+r-1 \\
r-1
\end{array}\right) \leq \frac{M 2^{r}}{(r-1) !} \frac{k^{r}}{2^{k}}
$$

Then choose $k_{0} \geq r$ such that $\frac{k^{r}}{\sqrt{2}^{k}} \leq 1$ forall $k \geq k_{0}$. Therefore

$$
\forall \mathbf{t}^{\prime} \in B(0, \varepsilon), \forall k \geq k_{0} \quad\left|W_{k}\left(\mathbf{t}^{\prime}\right)\right| \leq \frac{M 2^{r}}{(r-1) !} \frac{1}{\sqrt{2}^{k}}
$$

and

$$
\forall \mathbf{t}^{\prime} \in B(0, \varepsilon), \forall k \geq k_{0} \quad\left|\sum_{l \geq k} W_{l}\left(\mathbf{t}^{\prime}\right)\right| \leq \frac{M 2^{r}}{(r-1) !} \frac{\sqrt{2}}{\sqrt{2}-1} \frac{1}{\sqrt{2}^{k}}=C \frac{1}{\sqrt{2}^{k}}
$$

(7) Now we want to determine the computable number $z(\mathbf{q})=w(\mathbf{q}-\mathbf{t})$ for a given choice of $\mathbf{q} \in B(\mathbf{t}, \varepsilon) \cap(\mathbb{Q}+i \mathbb{Q})^{r}$. This number is one of the roots of $P(\mathbf{q}, z)$. 
These roots are computable numbers and so we can bound from below all the differences between each two of them: let $\delta \in \mathbb{Q}_{>0}$ be such a bound.

By the previous step one can compute an integer $k$ such that

$$
\forall \mathbf{t}^{\prime} \in B(0, \varepsilon),\left|\sum_{l \geq k} W_{l}\left(\mathbf{t}^{\prime}\right)\right| \leq C \frac{1}{\sqrt{2}^{k}} \leq \frac{\delta}{2} .
$$

So we can distinguish $z(\mathbf{q})$ from the other roots of $P(\mathbf{q}, z)$.

(8) Choose $\mathbf{q} \in(\mathbb{Q}+i \mathbb{Q})^{r}$ such that

(i) $\mathbf{q}$ is not in the discriminant locus of $P(t, z)$ seen as a polynomial in $z$,

(ii) $\|\mathbf{q}-\mathbf{t}\|<\varepsilon$,

(iii) $e_{j}\left(g_{i, \alpha}(\mathbf{q}, z(\mathbf{q}))\right) \neq 0$.

The first condition is insured by the choice of $\varepsilon$ in (5).

In order to check that $e_{j}\left(g_{i, \alpha}(\mathbf{q}, z(\mathbf{q}))\right) \neq 0$, one only has to choose $\mathbf{q}$ such that $\|\mathbf{t}-\mathbf{q}\|$ is small enough and this can be done effectively. Indeed the $e_{j}\left(g_{i, \alpha}\right)$ are rational functions in the $t_{i}$ and $z$, thus we can effectively bound the variations of $e_{j}$ locally around $\mathbf{t}$.

(9) Then we evaluate the $g_{i, a}(t, z(t))$ at $(\mathbf{q}, z(\mathbf{q}))$. We denote these values by $g_{i, \alpha}^{\prime}$ and we define the polynomials

$$
g_{i}^{\prime}:=\sum_{\alpha \in \mathbb{N}^{n}} g_{i, \alpha}^{\prime} x^{\alpha} .
$$

6.3. Generalized discriminants. We follow Appendix IV of Wh72, BPRbook or Roy06. The generalized discriminants, or subdiscriminants, $\Delta_{l}$ of a polynomial

$$
f=x^{d}+\sum_{j=1}^{d} b_{j} x^{d-j}
$$

can be defined as follows:

Let $\xi_{1}, \ldots, \xi_{d}$ be the roots of $f$ (with multiplicities), and set $s_{i}=\sum_{k=1}^{d} \xi_{k}^{i}$ for every $i \in \mathbb{N}$. Then

$$
\Delta_{d+1-l}=\left|\begin{array}{cccc}
s_{0} & s_{1} & \cdots & s_{l-1} \\
s_{1} & s_{2} & \cdots & s_{l} \\
\cdots & \cdots & \cdots & \cdots \\
s_{l-1} & s_{l} & \cdots & s_{2 l-2}
\end{array}\right| .
$$

Thus $\Delta_{1}$ is the classical discriminant of $f$. The polynomials $\Delta_{l}$ may be effectively computed in term of the $s_{i}$, and those can be effectively computed in terms of the $b_{i}$. The polynomial $f$ admits exactly $k$ distinct complex roots if and only if $\Delta_{1}=\cdots=\Delta_{d-k}=0$ and $\Delta_{d-k+1} \neq 0$.

\section{REFERENCES}

[BPRbook] S. Basu, R. Pollack, and M.-F. Roy, Algorithms in real algebraic geometry, vol. 10 of Algorithms and Computation in Mathematics, Springer-Verlag, Berlin, second ed., 2006. [BM90] E. Bierstone, P. D. Milman, Arc-analytic functions, Invent. math. 101 (1990), 411-424. [BPR17] M. Bilski, A. Parusiński, G. Rond, Local topological algebraicity of analytic function germs, J. Algebraic Geom., 26, (2017), 177-197.

[BCR] J. Bochnak, M. Coste, M.-F. Roy, Real Algebraic Geometry, Springer-Verlag, Berlin Heidelberg 1998. 
[BM95] L. P. Bos, P. D. Milman, Sobolev-Gagliardo-Nirenberg and Markov type inequalities on subanalytic domains, Geom. Funct. Anal., 5, (1995), no. 6, 853-923.

[CLO07] D. Cox, J. Little, D. O'Shea, Ideals, Varieties, and Algorithms, An introduction to computational algebraic geometry and commutative algebra, Springer, New York, 2007.

[DGY96] J.-P. Dedieu, X. Gourdon, J.-C. Yakoubsohn, Computing the distance from a point to an algebraic hypersurface, The mathematics of numerical analysis (Park City, UT, 1995), 285-293, Lectures in Appl. Math., 32, Amer. Math. Soc., Providence, RI, 1996.

[GP08] M. Greuel, G. Pfister, A SINGULAR introduction to commutative algebra, Springer, Berlin, 2008.

[H80] R. Hardt, Semi-algebraic local-triviality in semi-algebraic mappings. Amer. J. Math. 102 (1980), no. 2, 291-302.

[Kuo85] T.-C. Kuo, On classification of real singularities, Invent. math. 82 (1985), 257-262.

[Ku88] K. Kurdyka, Ensembles semi-algébriques symétriques par arcs, Math. Ann. 282 (1988), 445-462.

[KK18] W. Kucharz, K. Kurdyka, From continuous ration to regulous functions, Proc. Int. Cong. of Math. - 2018 Rio de Janeiro, Vol 1 (715-744).

[KP08] K. Kurdyka, A. Parusiński, Arc-symmetric Sets and Arc-analytic Mappings, dans "Arc Spaces and Additive Invariants in Real Algebraic Geometry", Proceedings of Winter School "Real algebraic and Analytic Geometry and Motivic Integration", Aussois 2003, eds. M. Coste, K. Kurdyka, A. Parusiński, Panoramas et Synthèses 24, 2008, 33-68.

[L95] L. Lempert, Algebraic approximations in analytic geometry, Invent. Math., 121 (1995), pp. 335-353.

[MP2011] C. McCrory, A. Parusiński, The weight filtration for real algebraic varieties, MSRI Publications, 18 "Topology of Stratified Spaces" (2011), 121-160

[Mo84] T. Mostowski, Topological equivalence between analytic and algebraic sets, Bull. Polish Acad. Sci. Math., 32, (1984), no. 7-8, 393-400.

[PP17] L. Păunescu, A. Parusiński, Arc-wise analytic stratification, Whitney fibering conjecture and Zariski equisingularity, Adv. Math., 309, (2017), 254-305.

[RD84] P. Ribenboim, L. Van den Dries,, The absolute Galois group of a rational function field in characteristic zero is a semi-direct product, Can. Math. Bull., 27, (1984), 313-315.

[Ri54] H. G. Rice, Recursive real numbers, Proc. Amer. Math. Soc., 5, (1954), 784-791.

[Ro] G. Rond, Local topological algebraicity with algebraic coefficients of analytic sets or functions, Algebra Number Theory, 12, (2018), no. 5, 1215-1231.

[Roy06] M.-F. Roy, Subdiscriminant of symmetric matrices are sums of squares, Mathematics, Algorithms, Proofs, volume 05021 of Dagstuhl Seminar Proceedings, Internationales Begegnungs und Forschungszentrum für Informatik (IBFI), Schloss Dagstuhl, Germany, (2005).

[Te90] B. Teissier, Un exemple de classe d'équisingularité irrationnelle, C. R. Acad. Sci. Paris Sér. I Math., 311, (1990), no. 2, 111-113.

[Tu36] A. M. Turing, On Computable Numbers, with an Application to the Entscheidungsproblem, Proc. London Math. Soc. (2), 42, (1936), no. 3, 230-265.

[Va72] A. N. Varchenko, Theorems on the topological equisingularity of families of algebraic varieties and families of polynomial mappings, Math. USSR Izviestija, 6, (1972), 949-1008.

[Va73] A. N. Varchenko, The relation between topological and algebro-geometric equisingularities according to Zariski. Funkcional. Anal. Appl. 7 (1973), 87-90.

[Va75] A. N. Varchenko, Algebro-geometrical equisingularity and local topological classification of smooth mappings. Proceedings of the International Congress of Mathematicians (Vancouver, B.C., 1974), Vol. 1, pp. 427-431. Canad. Math. Congress, Montreal, Que., 1975.

[Wh72] H. Whitney, Complex Analytic Varieties, Addison-Wesley Publ. Co., Reading, Massachusetts 1972 .

[Za71] O. Zariski, Some open questions in the theory of singularities, Bull. Amer. Math. Soc. $7 \mathbf{7}$ (1971) 481-491; Oscar Zariski: Collected Papers, Volume IV, MIT Press, 238-248.

E-mail address: adam.parusinski@unice.fr

Université Nice Sophia Antipolis, CNRS, LJAD, UMr 7351, 06108 Nice, France

E-mail address: guillaume.rond@univ-amu.fr

Aix Marseille Univ, CNRS, Centrale Marseille, I2M, Marseille, France 